\section{2cta 3 iologica Sibirica}

Journal of Biology

Founded in 2015
Altai State University

www.asu.ru

ISSN 2412-1908

\title{
Morphology and morphometry of the tibial organ of males of the genus Dolichopus Latreille, 1796 (Dolichopodidae, Diptera)
}

\author{
O.P. Negrobov, M.A. Chursina, O.V. Selivanova \\ Voronezh State University, Universitetskaya sq., 1, Voronezh 394006 Russia. \\ E-mail:negrobov@list.ru
}

Morphologic and morphometric characteristics of generic level of the tibial organ of males Dolichopus Latreille, 1796, were investigated. On the basis of examined characteristics the subgeneric variability were investigated and groups of superspecific level were allocated.

Key words: Dolichopus, Dolichopodidae, tibial organ, specific characters

\section{Морфология и морфометрия тибиального органа самцов Dolichopus Latreille, 1796 (Dolichopodidae, Diptera)}

\author{
О.П. Негробов, М.А. Чурсина, О.В. Селиванова
}

Воронежский государственный университет, Университетская пл., 1, Воронеж 394006 Россия

Изучены морфологические и морфометрические признаки видового уровня тибиального органа самцов рода Dolichopus Latreille, 1796. На основании изученных признаков была изучена межвидовая изменчивость и выделены группы надвидового уровня.

Ключевые слова: Dolichopus, Dolichopodidae, тибиальный орган, видовые признаки.

\section{Введение}

Сравнительно морфологические признаки составляют основу построения естественной системы и группировки таксонов. Исследования филогении семейства Dolichopodidae (Sinclair, Cumming, 2006; Wang et al., 2007; Germann et al., 2009; Negrobov et al., 2014) показали необходимость составления матрицы признаков, с использованием которой будут максимально достоверно рассчитаны статистические расстояния между таксонами одного ранга. Наибольшее количество признаков также позволит нивелировать субъективизм в оценке значимости отдельных морфологических данных.

Характеристики тибиального органа (цилиоратума) рода Dolichopus Latreille, 1796 до сих пор не использовались в систематике и изучении филогении рода. Цилиоратумом (cilioratium) (Steyskal, 1973) называется широкая или узкая щель с рядом щетинок на вентральной поверхности задней голени самцов рода Dolichopus, она располагается обычно ниже середины голени, в её дистальной части. Смирнов (1948а, 
Negrobov, O.P. et al. Morphology and morphometry of the tibial organ.... Acta Biologica Sibirica, 2018, 4(1), 46-51

1948b) впервые использует этот признак при описании видов и называет эту щель тибиальным органом. Введу того, что этот орган имеется только у самцов можно предположить, что это железы выделяющие половые феромоны.

Щетинки тибиального органа намного меньше, чем остальная хетотаксия голени (рис. 1). Форма щетинок отличается у разных видов. Они могут быть уплощенные, ланцетовидные, суженные апикально, или тонкие, удлинённые.

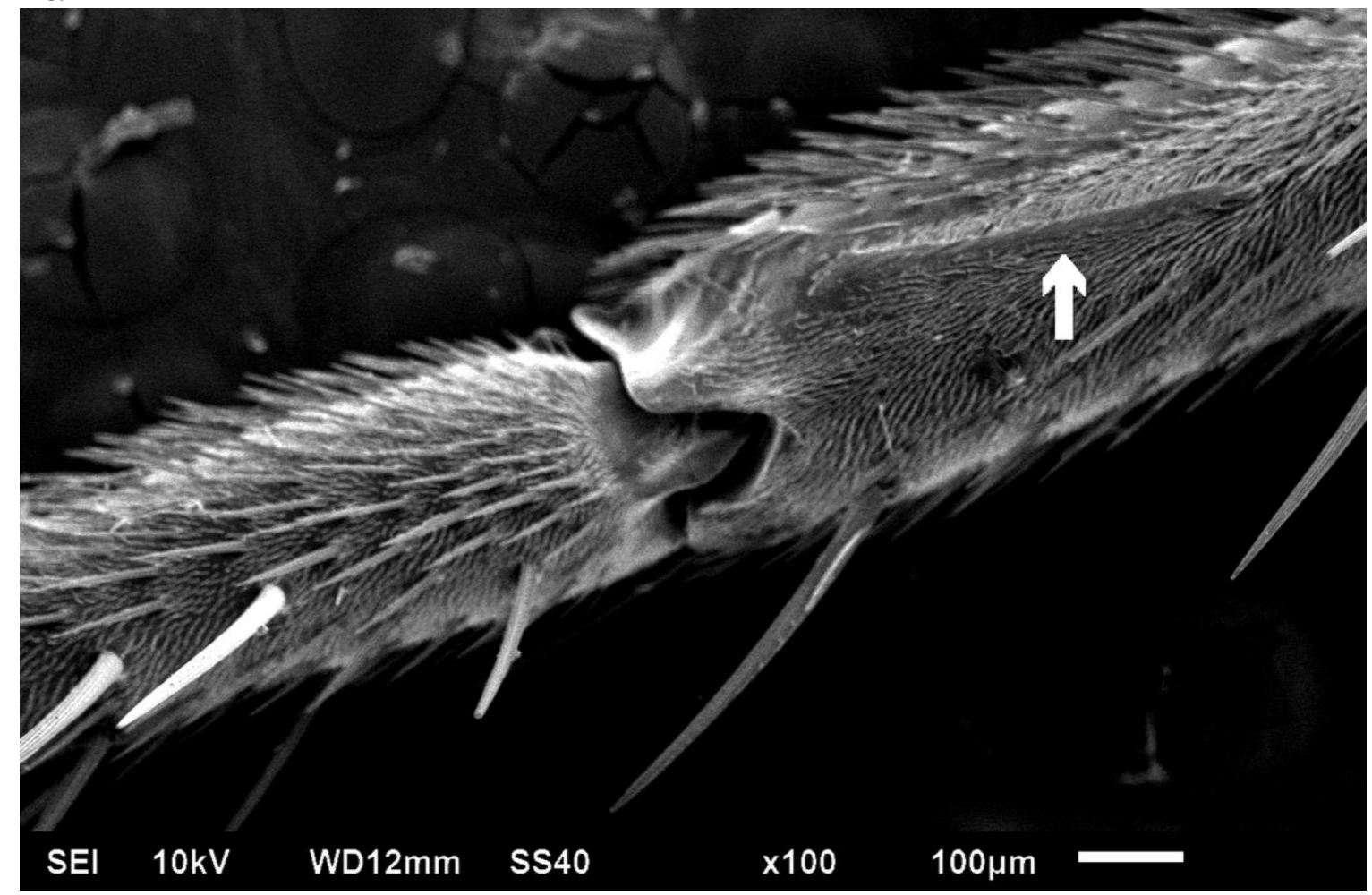

Рис. 1. СэМ изображение задней голени самца Dolichopus nataliae Stackelberg.

Тибиальный орган ряда видов расположен параллельно продольной оси голени, у других видов - под большим или меньшим углом к ней. По форме он может напоминать широкий желобок или может быть представлен гребнем щетинок (Andersson, 1977). Также различия наблюдаются в длине тибиального органа и его цветовых вариациях.

\section{Материал и методы исследований}

Целью работы было выявление систематических признаков в морфологии тибиального органа и их использование для выделения группировок подвидового уровня. Нами были рассмотрены 37 видов, принадлежащих к роду Dolichopus: D. acuticornis Fallen, 1823, D. agilis Meigen, 1824, D. altayensis Yang, 1998, D. amurensis Stackelberg, 1930, D. angustinennis Becker, 1922, D. bigeniculatus Parent, 1926, D. brevipennis Meigen, 1824, D. calceatus Parent, 1927, D. campestris Meigen, 1824, D. cilifemoratus Parent, 1926, D. claviger Stannius, 1831, D. galeatus Loew, 1871, D. jakutus Selivanova et Negrobov, 2011, D. jaxarticus Stackelberg, 1927, D. lepidus Zetterstedt, 1843, D. linearis Meigen, 1824, D. lineatocornis Zetterstedt, 1843, D. litorellus Zetterstedt, 1852, D. longicornis Stannius, $1831, D$. longitarsis Stannius, 1831, D. maculatus Bezzi, 1936, D. maculipennis Zetterstedt, 1843, D. nataliae Stackelberg, 1930, D. nigricornis Meigen, 1824, D. nigripes Fallen, 1823, D. nitidus Fallen, 1823, D. plumipes Fallen, 1823, D. plumitarsis Fallen, 1823, D. remipes Wahlberg, 1839, D. ringdahli Stackelberg, 1930, D. robustus Stackelberg, 1928, D. rupestris Haliday, 1833, D. simius Parent, 1927, D. simplex Meigen, 1824, D. trivialis Haliday, 1832, D. ungulatus Loew, 1850, D. ussuriensis Stackelberg, 1930, D. zernyi Parent, 1927.

Для анализа были использованы фотографии экземпляров Dolichopus из коллекции ВГУ, сделанные под бинокулярным микроскопом МБС-1 с помощью фотоаппарата Canon SX230. Фотографии органа Dolichopus nataliae производились на сканирующем электронном микроскопе JSM-6380LV (JEOL) Центра коллективного пользования научным оборудованием ВГУ. Измерения были выполнены по фотографиям в программе Adobe Illustrator. Для измерения были выбраны следующие показатели (рис. 2): диаметр голени (D), длина тибиального органа (I) и длина голени. Рассчитанные соотношения показателей обрабатывались статистически.

Рассмотрение видовой изменчивости не показало значимых результатов, и дальнейшие исследования были направлены на выделение групп надвидового уровня.

Для выделения групп видов использовались следующие признаки: 
Negrobov, O.P. et al. Morphology and morphometry of the tibial organ.... Acta Biologica Sibirica, 2018, 4(1), 46-51

1. Наклон тибиального органа относительно продольной оси голени: тибиальный орган практически параллелен продольной оси голени (0); тибиальный орган находится под углом около 20 градусов к продольной оси голени (1); тибиальный орган находится под углом около 45 градусов к продольной оси голени (2).

2. Отношение диаметра голени к длине тибиального органа: от 0,2 до 0,5 (0), от 0,6 до 1,3 (1); от 1,4 до 2,0 (2); от 2,1 до 3,0 (3).

3. Отношение длины голени к длине тибиального органа: от 0 до 4 (0), от 6 до 16 (1), от 17 до 26 (2).

4. Щетинки тибиального органа длинные, более $1 / 4$ длины тибиального органа (1). Щетинки короткие (0).

\section{Результаты и обсуждение}

В результате статистической обработки полученных данных в составе рода Dolichopus были выделены следующие группы видов (рис. 2).

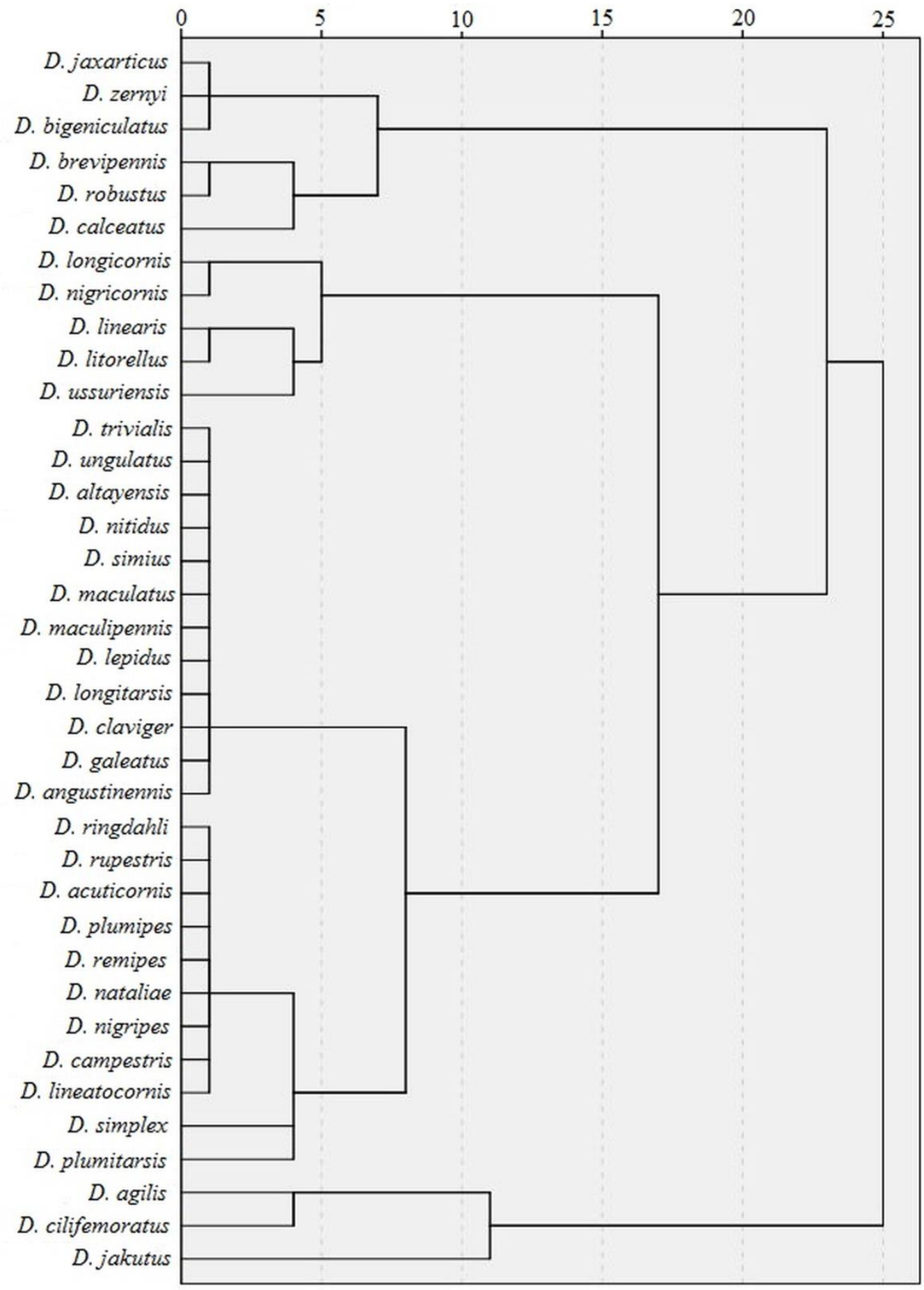

Pис. 2. Дендрограмма признаков морфологии тибиального органа видов Dolichopus. 
Группа 1 включает в себя виды Dolichopus, тибиальный орган которых значительной длины (1/4 длины голени и более), обычно параллелен продольной оси голени, без длинных щетинок (рис. 3-6), однако с явными щетинками у видов Dolichopus robustus. Щель тибиального органа может быть как относительно широкой (виды Dolichopus brevipennis, D. calceatus), так и очень тонкой (Dolichopus zernyi D. jaxarticus, D. bigeniculatus). У всех рассмотренных видов голень затемнена апикально, тибиальный орган светлый.
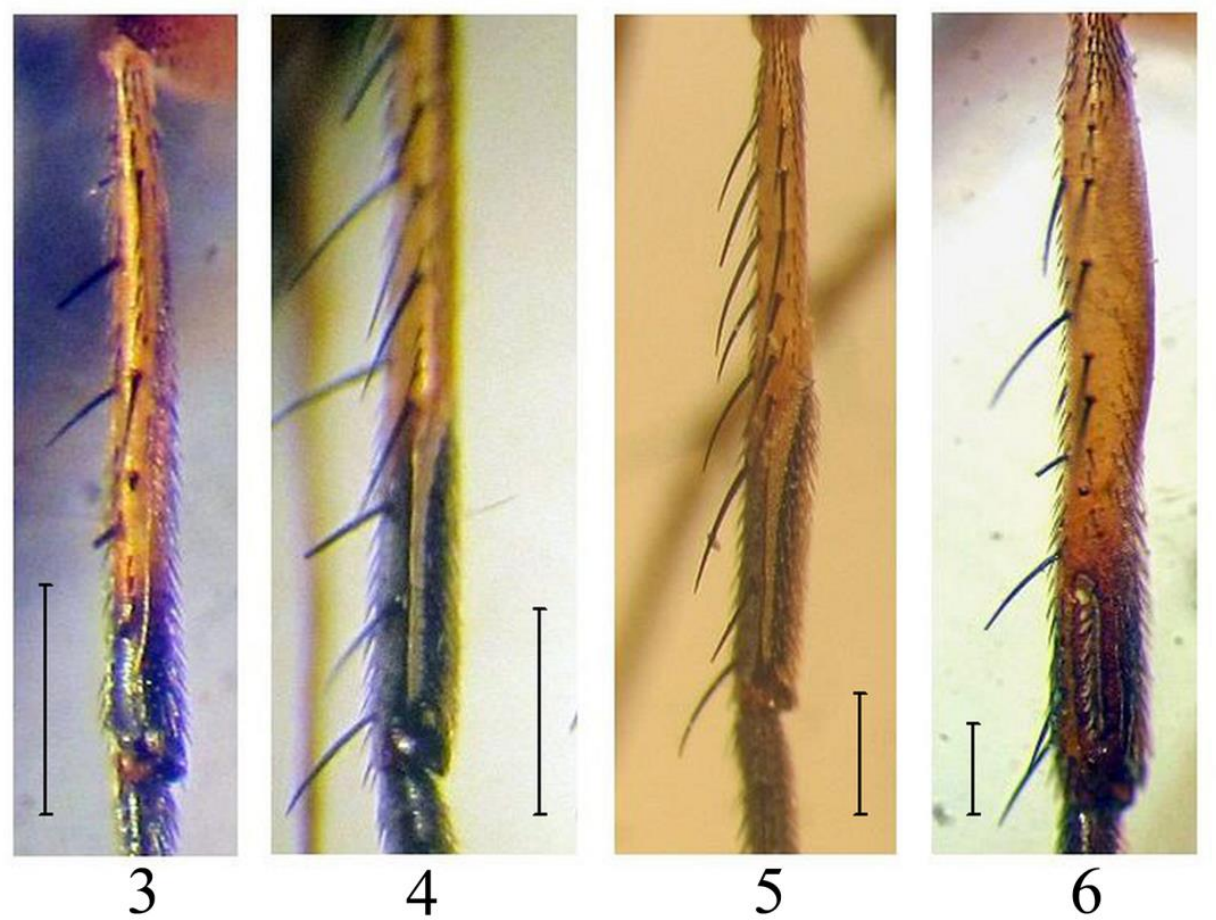

Pис. 3-6. Тибиальный орган самцов Dolichopus первой группы.

3. D. zernyi Parent; 4. D. brevipennis Meigen; 5. D. calceatus Parent; 6. D. robustus Stackelberg. Масштабная линейка: 0.5 мм.

Наиболее распространенная форма тибиального органа характерна для видов группы 2: тибиальный орган короче четверти голени, однако его длина превосходит диаметр голени или примерно равна ему. Тибиальный орган обычно располагается под углом $15-20^{\circ}$ по отношению к продольной оси голени, имеет светлые короткие щетинки. Данная группа включает виды Dolichopus altayensis, D. angustipennis, D. claviger, D. galeatus, D. lepidus, D. longitarsis, D. maculates, D. maculipennis, D. nitidus, D. trivialis, D. ungulatus.

Задняя голень рассмотренных видов затемнена апикально, и тибиальный орган полностью располагается на затемнённой части (рис. 7-9). Однако выделяется ряд видов (Dolichopus lepidus, D. altayensis, D. maculatus) с полностью чёрными задними голенями (рис. 10-12).

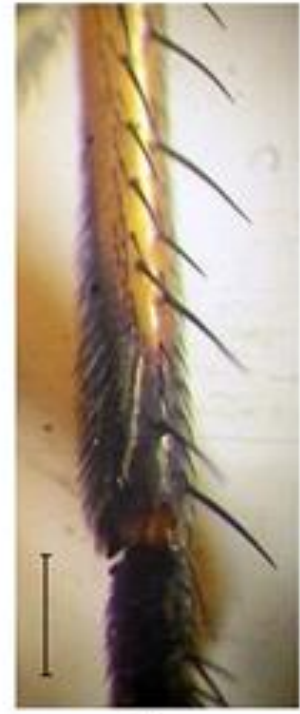

7

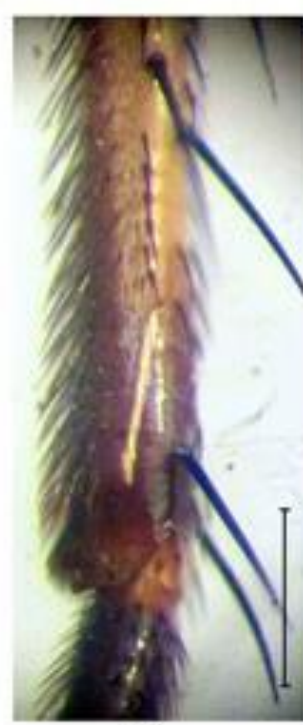

8

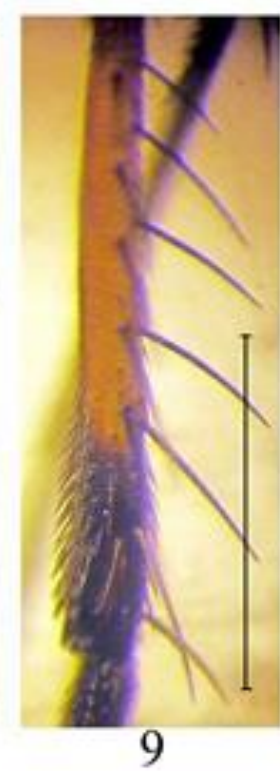

9

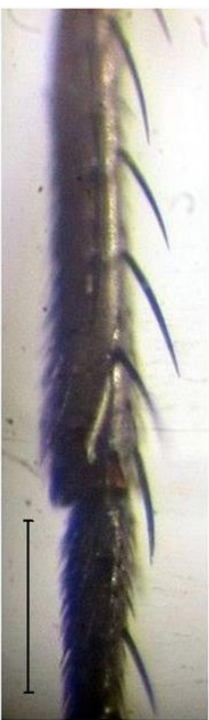

10

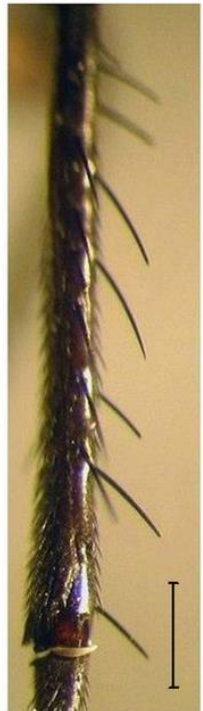

11

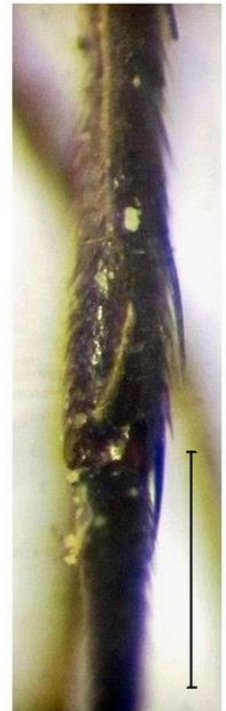

12

Рис. 7-12. Тибиальный орган самцов Dolichopus второй группы.

7. D. galeatus Loew; 8. D. ungulatus Linnaeus; 9. D. longitarsis Stannius; 10. D. lepidus Staeger; 11. D. altayensis Yang; 12. D. maculatus Parent. Масштабная линейка: 0.5 мм. 
Тибиальный орган у видов группы 3 короткий, его длина меньше диаметра голени, и значительно меньше её длины, обычно - с удлинёнными светлыми щетинками, располагается под углом 25-35 по отношению к продольной оси голени (рис. 13-16).

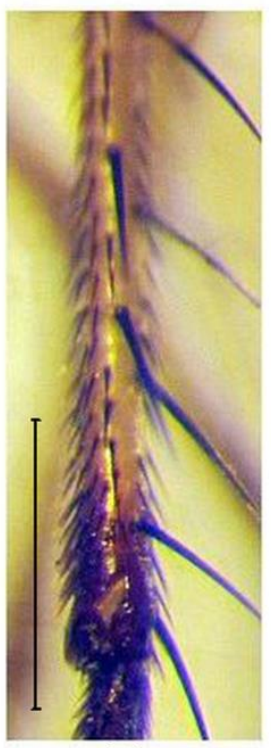

13

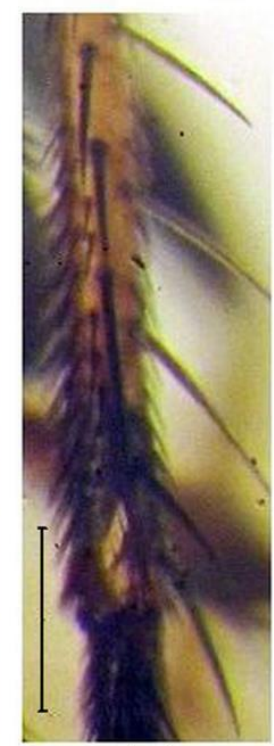

14

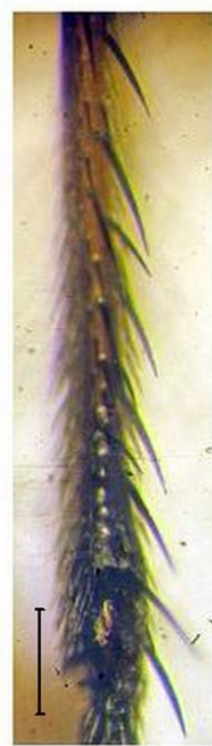

15

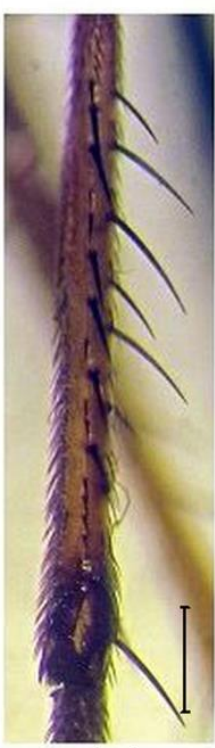

16

Рис. 13-16. Тибиальный орган самцов Dolichopus третьей группы.

13. D. acuticornis Wiedemann; 14. D. campestris Meigen; 15. D. remipes Wahlberg; 16. D. rupestris Haliday.

Масштабная линейка: 0.5 мм.

Группа 4. Отдельно следует рассматривать подгруппу, включающую виды Dolichopus longicornis, D. nigricornis, D. linearis, D. litorellus, D. ussuriensis. Данная группа характеризуется укороченным тибиальным органом с удлинёнными щетинками. Длина щетинок тибиального органа обычно составляет от 0,25 до 1,50 его длины (рис. 17-18). Щетинки тибиального органа во всех случаях светлые, в то время как голень затемнена апикально.

Тибиальный орган средней длины, расположенный в глубокой вырезке, находящейся под небольшим углом к продольной оси голени характерен для видов различных групп: Dolichopus cilifemoratus, D. plumitarsis, D. robustus, D. jakutus, D. cilifemoratus (рис. 19-21). Данный признак не использовался в качестве группообразующего, поскольку остальные морфологические характеристики тибиального органа перечисленных видов были различны.

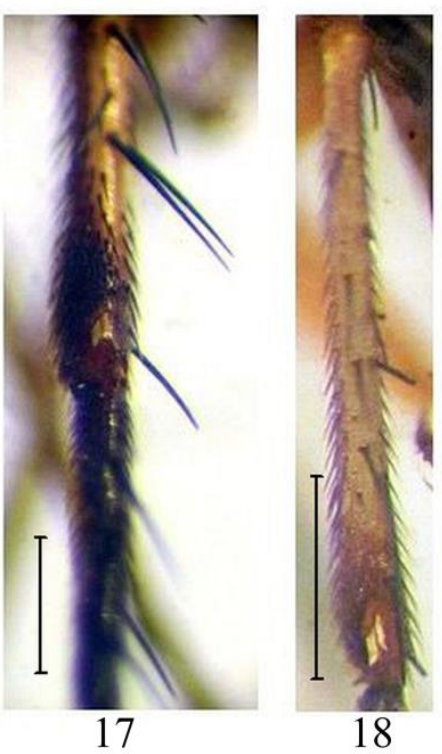

Рис. 17-18. Тибиальный орган самцов Dolichopus четвёртой группы.

17. D. ussuriensis Stackelberg; 18. D. Iongicornis Stannius. Масштабная линейка: 0.5 мм.

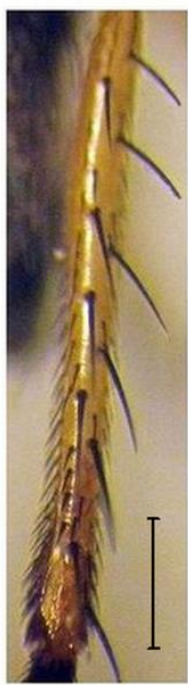

19

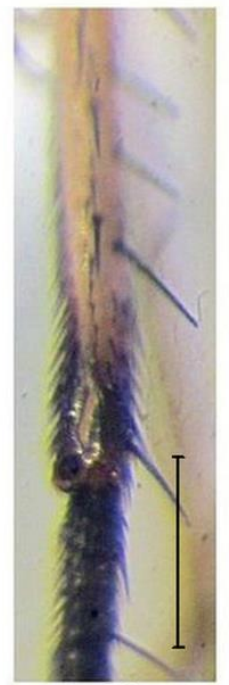

20

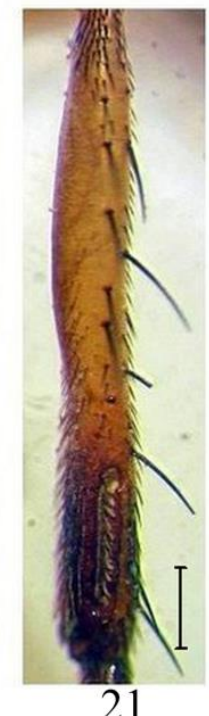

21
Рис. 19-21. Тибиальный орган самцов Dolichopus в виде желобка.

19. D. cilifemoratus Macquart; 20. D. plumitarsis Fallen; 21. D. robustus Stackelberg. Масштабная линейка: 0.5 мм. 
Negrobov, O.P. et al. Morphology and morphometry of the tibial organ.... Acta Biologica Sibirica, 2018, 4(1), 46-51

Исходя из рассмотренных данных, следует отметить, что морфология тибиального органа может быть использована для изучения системы и филогении рода Dolichopus наряду с признаками морфологии антенн, лица и хетотаксии ног. Дальнейшие исследования должны быть направлены на увеличение базы данных за счёт рассмотрения дополнительных видов, а также в случае необходимости, выделения дополнительных признаков морфологии, касающихся соотношения цветовых характеристик голени и тибиального органа, формы вырезки и её глубины.

\section{Благодарности}

Авторы выражают благодарность Центру коллективного пользования научным оборудованием Воронежского госуниверситета, на оборудовании которого были проведены исследования по анализу поверхности. Работа выполнена при поддержке гранта РФФИ № 14-04-00264.

\section{References}

Andersson H. (1977). Taxonomic and phylogenetic studies on Chloropidae (Diptera) with special reference to Old World genera. Entomologica Scandinavica (Suppl.). 8, 1-200.

Germann C., Pollet M., Tanner S., Backeljau T., Bernasconi M.V. (2009). Legs of deception: disagreement between molecular markers and morphology (Diptera, Dolichopodidae). Journal of Zoological Systematics and Evolutionary Research. 48, 238-247.

Sinclair B. J., Cumming, J. M. (2006). Morphology, higher-level phylogeny and classification of the Empidoidea. Zootaxa. 1180: 1-172.

Smirnov E.S. (1948 a). Materials for the Dolichopus Latr the Far East. I. Scientific and methodological notes of the Main Directorate for Reserves. 2, 223-229 (in Russian).

Smirnov E.S. (1948 b). Materials for the Dolichopus Latr the Far East. II. Scientific and methodological notes of the Main Directorate for Reserves.2, 230-241 (in Russian).

Steyskal G.C. (1973). The North American Species of Dolichopus Latreille, Group B (Diptera, Dolichopodidae). Journal of the Kansas Entomological Society. 46 (3), 347-359.

Wang M.Q., Zhu Y.J., Zhang L., Yang D. (2007). A phylogenetic analysis of Dolichopodidae based on morphological evidence (Diptera, Brachycera). Acta Zootaxonomia Sinica. 32 (2), 241-254.

\section{Citation:}

Negrobov, O.P., Chursina, M.A., Selivanova, O.V. (2018). Morphology and morphometry of the tibial organ of males of the genus Dolichopus Latreille, 1796 (Dolichopodidae, Diptera). Acta Biologica Sibirica, 4 (1), 46-51.

Submitted: 12.12.2017. Accepted: 20.02.2018

cross ref http://dx.doi.org/10.14258/abs.v4i1.3916 